Quasi-periodic vertical structures of dusty plasma crystals

This article has been downloaded from IOPscience. Please scroll down to see the full text article. 1999 Europhys. Lett. 46190

(http://iopscience.iop.org/0295-5075/46/2/190)

The Table of Contents and more related content is available

Download details:

IP Address: 140.112.113.225

The article was downloaded on 01/07/2009 at 11:54

Please note that terms and conditions apply. 


\title{
Quasi-periodic vertical structures of dusty plasma crystals
}

\author{
Tzinong Chiueh ${ }^{1,3}$ and D. Ming-Ting Kuo ${ }^{2}$ \\ 1 Physics Department, National Taiwan University - Taipei, Taiwan \\ 2 Physics Department, University of Illinois, Urbana-Champaign - IL 61801, USA \\ 3 Institute of Astronomy and Astrophysics, Academia Sinica - Taipei, Taiwan
}

(received 10 November 1998; accepted in final form 5 February 1999)

PACS. 52.40Hf - Plasma-wall interactions; boundary layer effects; plasma sheaths.

PACS. 52.35Mw- Nonlinear waves and nonlinear wave propagation (including parametric effects, mode coupling, ponderomotive effects, etc.).

PACS. $52.90+\mathrm{z}-$ Other topics in physics of plasmas and electric discharges (restricted to new topics in section 52).

\begin{abstract}
A semi-analytical model is proposed for understanding the quasi-periodic vertical structures in a plasma crystal, which are shown to be sustained by the combined effects of an ion flow, gravity and horizontal bonds of the dust particles. These structures are the manifestations of large-amplitude standing wake fields that lead to strong vertical bonds for the formation of an oriented hexagonal lattice. The orientational symmetry breaking is caused by a gravity-induced thick sheath, which can preferentially accelerate a supersonic ion flow in the vertical direction. The electron and streaming ion densities and the electric potential are found to be all in phase in the plasma crystal, whereas the dust density is 180 degrees out of phase. With each dust layer playing a similar role as the plasma wall, the Bohm sheath criterion is also found to be satisfied. Crystal melting may also be qualitatively described by the present model when the horizontal bonds of the dust particles are sufficiently weakened.
\end{abstract}

Introduction. - Recent experiments have remarkably demonstrated the possibility of crystallization in the dusty plasmas [1-4]. In an isotropic three-dimensional crystal, the stable lattices have been shown to energetically favor the face-center cubic (fcc) and body-center cubic (bcc) configurations $[5,6]$. It is therefore unexpected to find that some of these plasma crystals are truly three-dimensional and yet exhibit oriented hexagonal configurations, characteristic of the two-dimensional lattices, with the preferred axis usually pointing toward that of the gravity. Such a phenomenon can only occur when the vertical bonds of the dust particles are exceptionally strong, thereby breaking the three-dimensional orientation symmetry.

Investigations on the issue of vertical bonds in plasma crystals have been conducted in the past. It has been pointed out that the ion streaming motion, much like that in the plasma sheath, plays the key role for forming the strong vertical bonds [7-11]. The physics of the plasma sheath is well understood. It is the rapid loss of high-mobility electrons that creates the sheath electric field near the wall, which is sustained self-consistently by accelerating a supersonic flow of cold ions toward the wall. In the present context, the collection of dust 
particles may be regarded as a series of penetrable walls, which on the one hand allow the streaming ions to pass through and on the other hand become corrugated in response to the local electric field fluctuations in the plasma.

The following simulation results help establish a physical picture as to how the plasma crystal may arise. It has been found that the positively charged ions passing through a single dust particle can be slowed down behind the negatively charged dust particle and create a region of high ion density through excitation of a downstream wake field [7]. When the ions are streaming through two dust particles which are aligned streamwise, it is further found that these ions are focused in between the two dust particles, creating a cloud of positive space charge in attracting the two negatively charged dust particles [8-11]. Linear perturbation calculations with the self-consistent plasma response in a pre-arranged dust lattice also indicate a similar trend [11]. On the other hand, when the ions vertically stream through two dust particles which are aligned horizontally, the downstream wake field can be extendedly distributed in the horizontal directions, and hence can also serve to bind the dust particles transversely. The horizontal separation of the dust particles is found to self-regulate in such a way that the two particles are bound by a downstream ion cloud located in between them. Such horizontal bonds persist even in the plasma lattice [11].

In spite of the above careful investigations, in which the ion flow is prescribed, little attention has been paid as to why it should be the vertical direction along which the ion flow is particularly dominant, thereby defining the symmetry-breaking direction. In this report, we propose a simple analytical model for understanding the formation of oriented hexagonal plasma crystal. This model not only captures the essential physics of the abovementioned numerical studies but also yields a gravity-induced presheath that defines the preferential direction. Moreover, unlike these previous studies, which either deal with a small number of dust particles $[9,10]$ or regard the plasma response as linear perturbations [11], the vertical bond strength can be determined in a self-consistent way in the present model. Although the complicated three-dimensional potential structures and ion orbits found in the previous numerical studies are indeed very revealing, with a proper averaging procedure, these complicated details can be smoothed away. The reduction of complexity does not lose the key physics for the excitation of wake fields but only to allow the problem to be analyzed with its full nonlinearity. The solutions so obtained describe how the ion, electrons and dust must be distributed vertically in order to create the strong vertical bonds in the plasma crystal.

Parameter ranges and the theoretical model. - The dust particles in the experiments can be of various sizes [1], ranging from few to several $\mu \mathrm{m}$, and show vertical stratification, with heavy particles sunk at the bottom of the lattice. The density of a closely packed dust particle is about few $\mathrm{g} / \mathrm{cm}^{3}$; however, when taken into account the fact that the dust particles grow through aggregation and may not be closely packed, we let the mean density of a particle be about $1 \mathrm{~g} / \mathrm{cm}^{3}$. The mean mass for a medium-size particle, i.e., $5 \mu \mathrm{m}$, is about $M_{\mathrm{d}} \sim 3 \times 10^{-10} \mathrm{~g}$. With a crystal of about 10 vertical monolayers of $100 \mu \mathrm{m}$ layer spacing, the dust gravitational potential energy density is estimated to be $3 \times 10^{-2} \mathrm{erg} / \mathrm{cm}^{3}$. On the other hand, the electron pressure for the eV electrons of number density $10^{10} \mathrm{~cm}^{-3}$ is about $2 \times 10^{-2} \mathrm{erg} / \mathrm{cm}^{3}$. This estimate shows that gravity can be sufficiently strong to create significant long-range vertical stratification over the distance of 10 layer spacings, but is relatively weak in the force balance on the length scale of one layer spacing. This finding is important, in that though weak on the lattice-spacing scale, the gravity can yield a large-scale presheath, which preferentially accelerates an ion flow in the vertical direction and breaks the orientational symmetry.

We may also estimate the charge of the dust particles. Let the effective negative charge of the dust particle include that of the neutralizing trapped ions around the dust; the total dust charge is few times smaller than the total electron charge. Given the averaged dust density 
$3 \times 10^{5} \mathrm{~cm}^{-3}$, the dust charge is hence about $Q_{\mathrm{d}} \sim-10^{4} e$, where $e$ is the absolute value of the electron charge. The electron Debye length $\lambda_{\mathrm{D}}$ can be estimated from the above-quoted electron temperature and density, and $\lambda_{\mathrm{D}}$ turns out to be $50 \mu \mathrm{m}$, few times smaller than the observed lattice spacing.

In competing against the plasma pressure force, which does not favor crystallization in the plasma, the individual dust particles in the plasma crystals are actually bound by the vertical and horizontal attractive fields mediated by the properly located ion clouds in between dust layers. The average vertical bond strength can be described by the horizontally averaged vertical electric field, which is to be determined. The horizontal bond strength will be parameterized in the present model, and the vertical bond strength will turn out to sensitively depend on the horizontal bond strength. In fact, in this one-dimensional model, the contribution of the horizontal bonds to the vertical force balance must be explicitly accounted for. This is because the dust particles can no longer behave as an isotropic gas, but as an anisotropic fluid that has a shear stress, much like a magnetofluid. Thus, the collection of dust particles may be regarded as consisting of many layers of penetrable membrane thread by horizontal bonds, and the membrane has surface tension in rectifying the surface corrugation. Without such a restoring tension force, the surface corrugation, arising from the bombardments of electrons and ions, can manifest itself as an increasingly broadened dust density profile in this one-dimensional model. On the contrary, the surface tension tends to sharpen the diffuse dust density profile. While the surface corrugation can result from the electron pressure force, how may the self-focusing effect of surface tension be modeled in the one-dimensional model?

In modeling the effect of surface tension, we note that the strength of tension is proportional to the horizontal bond strength and hence to the first power of the local dust charge density $Q_{\mathrm{d}} n_{\mathrm{d}}$. We may hence model the focusing surface tension by a negative dust pressure force, which takes the form $-\mathrm{d}\left(\psi Q_{\mathrm{d}} n_{\mathrm{d}}\right) / \mathrm{d} z$, with $\psi$ being a negative constant. Here, $\psi\left|Q_{\mathrm{d}}\right|$ represents an effective horizontal binding energy per dust particle. Such a representation for the averaged surface tension is the simplest choice that captures the correct sign and the linear dependency on $n_{\mathrm{d}}$. (Any higher-order corrections must involve higher odd derivatives of $n_{\mathrm{d}}$ to ensure no irreversible effects to be introduced.)

Thus, the dust vertical force balance now reads

$$
0=Q_{\mathrm{d}} n_{\mathrm{d}} \frac{\mathrm{d} \phi}{\mathrm{d} z}-\psi \frac{\mathrm{d} Q_{\mathrm{d}} n_{\mathrm{d}}}{\mathrm{d} z}-M_{\mathrm{d}} n_{\mathrm{d}} g,
$$

where the dust gravity has been accounted for and $\phi$ is the horizontally averaged electric potential. Equation (1) can be immediately integrated to yield $Q_{\mathrm{d}} n_{\mathrm{d}}=Q_{\mathrm{d} 0} n_{\mathrm{d} 0} \exp [(\phi / \psi)-$ $\left.\left(M_{\mathrm{d}} g /\left|Q_{\mathrm{d}}\right|\right) z\right]$, where the subscript " 0 " denotes quantities evaluated in the plasma bulk, in which the dust density is small. In obtaining this expression, we have assumed $M_{\mathrm{d}}(z) / Q_{\mathrm{d}}(z)$ to be a constant because both $Q_{\mathrm{d}}(z)$ and $M_{\mathrm{d}}(z)$ are decreasing functions of height according to the experiments, and their ratio can be a weak function of height. Note that without the contribution from the surface tension, $\phi$ is soluble from eq. (1) and the solution defines the underlying electric potential of a large-scale sheath that extends well into the plasma bulk.

On the other hand, the ions are at about the room temperature and thus cold; therefore their momentum equation reads

$$
m_{i} \frac{\mathrm{d}}{\mathrm{d} z}\left(n_{i} v_{i}^{2}\right)=-n_{i} e \frac{\mathrm{d} \phi}{\mathrm{d} z} .
$$

In fact, the key effects of the complicated three-dimensional ion motions that exhibit ion focusing are retained in this averaged equation and manifest themselves as slowing-down of vertical ion motions behind the dust layer due to the electric attraction by dust particles. What has been neglected in eq. (2) from the ion motions is the centrifugal force of curved 
ion orbits. Since the streaming motion of ions is in the vertical direction, the bending of orbit yields only a horizontal centrifugal force. For example, at a moderate ion Mach number $M \sim 2$ and given the above experimental parameters, an orbit estimate shows that the kinetic energy of the transverse velocity for most ions (> 80 percent) is far smaller than that of the streaming velocity, and hence the contribution of the centrifugal force to the vertical force balance is negligible in comparison with the inertial force on the left-hand side of eq. (2). The ion continuity equation demands a constant vertical ion flux $f_{i}$, where $f_{i}=n_{i} v_{i}$. Equation (2) can also be integrated with the help of the constant $f_{i}$. It yields $n_{i}=f_{i}\left[\left(2 e / m_{i}\right)\left(\phi_{1}-\phi\right)\right]^{-1 / 2}$, where $e \phi_{1}$ is an integration constant, equal to $\left(m_{i} / 2\right)\left(f_{i} / n_{0}\right)^{2}$ if we let the plasma potential in the plasma bulk be zero. Finally, the eV electron temperature warrants that the electron pressure force be much higher than the electron inertial force and we may ignore the electron inertia. The electron momentum equation can thus be integrated once to yield the isothermal electron density, $n_{\mathrm{e}}=n_{0} e^{e \phi / T}$.

Substituting the dust, ion and electron densities into Poisson's equation, we arrive at the governing equation for the large-amplitude, horizontally averaged wake fields,

$$
\frac{\mathrm{d}^{2} \chi}{\mathrm{d} \xi^{2}}=\left[e^{\chi}-\left(1-\frac{2 \chi}{M^{2}}\right)^{-1 / 2}+\beta e^{\left.(\chi / \kappa)-\left(\xi-\xi_{0}\right) / L\right)}\right],
$$

where $\chi \equiv e \phi / T_{\mathrm{e}}, \xi \equiv-z / \lambda_{\mathrm{D}}$ with the Debye length $\lambda_{\mathrm{D}} \equiv \sqrt{T_{\mathrm{e}} / 4 \pi n_{0} e^{2}}$, the ion-flow Mach number $M \equiv\left(f_{\mathrm{i}} / n_{0}\right) \sqrt{m_{\mathrm{i}} / T_{\mathrm{e}}}, \beta \equiv\left|Q_{\mathrm{d} 0}\right| n_{\mathrm{d} 0} / e n_{0}, \kappa \equiv e \psi / T_{\mathrm{e}}$, and the scale height of gravitational stratification in unit of the Debye length $L \equiv\left|Q_{\mathrm{d}}\right| T_{\mathrm{e}} /\left(e M_{\mathrm{d}} g \lambda_{\mathrm{D}}\right)$. We now give the values of these dimensionless parameters appropriate to describe the experiments [1]. Since most dust particles are sunk in the lattice region and nearly absent in the bulk plasma, the parameter $\beta$ should assume a value much smaller than unity. The Mach number $M \sim O(1)$, and the scale length $L \sim 20$. What remains unknown a priori is the value of $\kappa$, which is a measure of the horizontal binding energy in unity of $T_{\mathrm{e}}$. It turns out that the ratio of the total dust charge relative to the total electron charge in our solution depends on $\kappa$, and we may compare the estimated dust charge with the solved dust charge to fix $\kappa$ at the end of our calculation. Generally, we have $\kappa \sim O\left(10^{-1}\right)$.

Gravitationally stratified quasi-periodic lattice. - To gain an intuition as to how the solution should behave, we may first ignore the $z$-dependence of the factor $\exp \left[\left(\xi_{0}-\xi\right) / L\right]$ in the last term of eq. (3) since $L \gg 1$ and absorb this factor into the coefficient $\beta$. This approximation has an analogy in the particle dynamics, where one considers the adiabatic change of a potential. We integrate eq. (3) once to obtain

$$
\frac{1}{2}\left(\frac{\mathrm{d} \chi}{\mathrm{d} \xi}\right)^{2}+U(\chi)=H
$$

where $H$ is the pseudo-energy and the pseudo-potential $U$ is

$$
U(\chi) \equiv-\left[e^{\chi}+M^{2}\left(1-\frac{2 \chi}{M^{2}}\right)^{1 / 2}\right]+\left(\kappa \beta^{\prime}\right) e^{-\chi / \kappa} .
$$

Here, the effective $\beta^{\prime} \equiv \beta \times \exp \left[-\left(\xi-\xi_{0}\right) / L\right]$. To make the solution behavior transparent, we can take a further limit that $\beta^{\prime} \rightarrow 0$. For a small $\kappa$, the contribution from the dust particles to the potential $U$, the third term in eq. (5), resembles a sharply rising potential wall located at $\chi \approx-\kappa\left|\ln \left(\kappa \beta^{\prime}\right)\right|$. (See fig. 1.) As soon as the value of $\chi$ reaches this critical value, the "force" contribution from the dust rises sharply and abruptly reflects the solution trajectory back to 


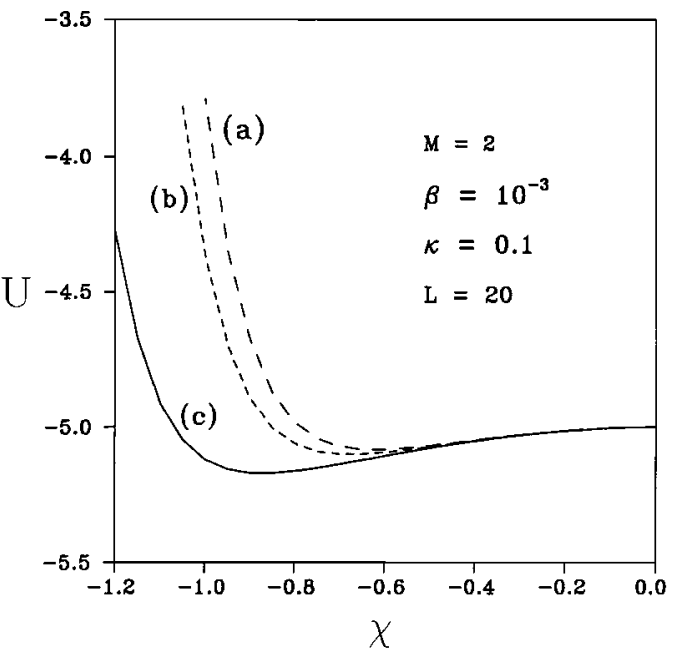

Fig. 1

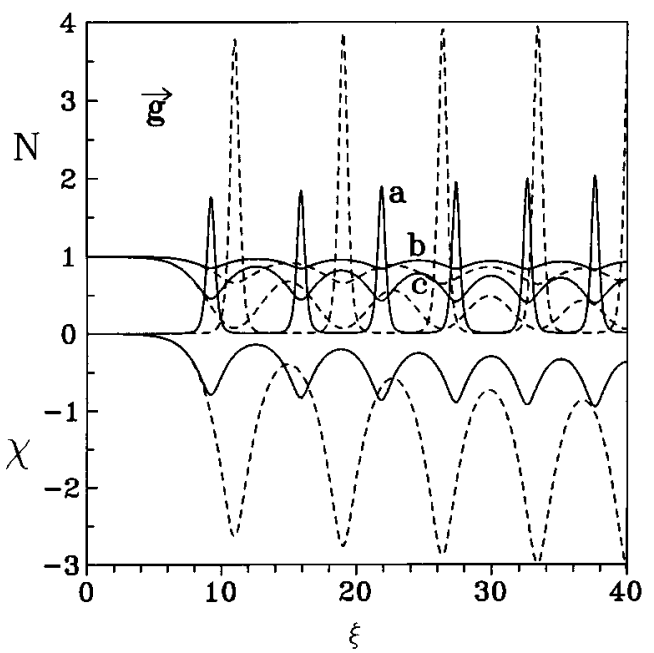

Fig. 2

Fig. 1. - The adiabatic change of the pseudo-potentials $U(\chi)$ at three decreasing heights: $\xi \equiv z / \lambda_{\mathrm{D}}=$ -8 (dashed line), -18 (dotted line), -28 (solid line). The parameters chosen are $M=2, \kappa=0.1$, $\beta=10^{-3}$ and $L=20$.

Fig. 2. - The solid lines are the (a) dust, (b) ion, (c) electron charge densities and the electric potential $\chi$ for the same parameters given in fig. 1. The dotted lines are for those densities and electric potential at $\kappa=0.3$, with other parameters unchanged.

$\chi=0$. Moreover, when $M>1, \chi=0$ is an unstable equilibrium point in the $\beta^{\prime} \rightarrow 0$ limit. Thus, in the plasma bulk, $\chi \rightarrow 0$ sits on the top of a potential hill for a supersonic ion flow, and must roll to the negative side in order to approach the negatively charged dust region.

We may immediately picture how the solutions should behave. First, the trajectories must be trapped within the region of negative $\chi$. Second, when the gravity is turned on, the pseudo-potential $U$ becomes ever-increasingly deepened in an adiabatic fashion due to the slow decrease of $\beta^{\prime}$, and the trajectories will drift away from the equilibrium $\chi=0$ after few oscillations and become deeply trapped within the decreasing potential well $U$. Note that since $\chi$ is negative, the solution can never be singular (cf. eq. (5)). Plotted in fig. 1 are three increasingly deepened pseudo-potentials $U$ at three decreasing $\xi$. Notice that the locations of the "reflecting walls" at negative $\chi$ hardly move at all. Deep into the bulk plasma, where $\chi=0$, the ions are gradually accelerated toward the bottom wall, and one expects that the solution should take a relatively large distance to depart from this unstable equilibrium and rolls down the potential well. The subsequent oscillations are expected to proceed faster as the lower heights are reached and as the solution trajectory drifts away from $\chi=0$. Indeed, the actual solution obtained from direct integration of eq. (3) shows the expected behaviors. Plotted in fig. 2 are the solution trajectories (solid lines) for $M=2, \beta=10^{-3}, \kappa=0.1$ and $L=20$. The electric potential, ion density and electron density oscillate in phase, but the dust density oscillates 180 degrees out of phase.

At smaller Mach numbers, both the lattice spacing and amplitude of oscillations are found to become smaller; no qualitative difference is found as long as $M>1$. The sharp peaks in the dust density arise mainly from the small $\beta^{\prime}$ and have little to do with both $\kappa$ and $M$. This is seen from the dotted lines in fig. 2, which show the persistence of sharp peaks when $\kappa=0.3$, 
with other parameters held fixed. The total charge ratio between the dust and electrons increases by a factor of 5 when $\kappa$ is raised from 0.1 to 0.3 . A similar trend also occurs for an increasing Mach number $M$. On the other hand, keeping $M$ and dust-to-electron total charge ratio fixed, we find that to maintain the same lattice spacing, a decreasing $\kappa$ must be accompanied by an increasing $\beta$, which gives rise to an increasingly broad dust density profile. This point is central to the discussion of crystal melting given below.

Note also that there exists a secular decrease of the electric potential on approaching the bottom in fig. 2. This trend results from the gravitational stratification of dust particles that forces the bottom to charge more negatively. Such a large-scale sheath can extend well into the interior of the plasma and draws an ion flow preferentially in the vertical direction. The orientational symmetry breaking in the plasma crystal is therefore originated from the dust gravity, although the gravity is relatively weak in comparison with the lattice electric forces.

In retrospect, we may examine whether the negative pressure modeled to represent the layer tension may create any spurious external force. We integrate the negative pressure force and we find that the total momentum flux contributed by it is proportional to the difference of the dust density in the bulk plasma and at the wall. Since in both regions the dust densities are negligibly small, the negative pressure force contributes no net momentum flux and indeed represents an internal force.

Discussions. - In sum, we report a simple, semi-analytical model for understanding how the strong vertical bonds may arise in a dusty plasma crystal. This model incorporates the ion streaming motion toward the bottom plasma wall, the dust gravity and the tension of dust layers. Among these factors, it is the combination of the ion flow and layer tension that play the key roles in creating the large-amplitude wake pattern that forms the strong vertical bonds. Although the effect of gravity is relatively minor on the scale of a lattice spacing, the overall stratification of the dust particles produced by the gravity nevertheless creates in the system a large electric potential drop, which induces the intense downward ion flow. These nonlinear wake patterns are the horizontal average of the much more complicated, three-dimensional wakes similar to those given in a previous study adopting the linear response theory [11]. To make further comparisons, we find that our plasma potential is charged more positively in between dust layers than at the dust layer, indicative of an excess of streaming ions in between the layers and suggesting the operation of ion focusing reported earlier [8-10]. The minimum density of the streaming ions occurs at the dust layer, also reflecting the acceleration of the average ion flows on approaching the dust layer. (In reality, there should be a population of trapped ions around each dust particle; however, in the present model we regard them as an integrated component of the dust particle.) The effective horizontal binding potential $\psi$ is found to be considerably smaller than the electron thermal energy $T_{\mathrm{e}}$ in order to yield the estimated dust charge. Given this small value of $\psi$, the vertical cage potential $\Delta \phi$ is found to be comparable to, or even larger than, $T_{\mathrm{e}}$ (cf. fig. 2), suggestive of the much stronger vertical bonds than the horizontal bonds in the plasma crystal. This finding arises as a natural consequence of the present model when a moderately strong ion flow (e.g., $M \sim 2$ ) is present. Indeed, it is under these conditions that the vertical axis of the plasma crystal can be so rigid as to lead the lattice to assume an oriented hexagonal configuration.

Along this line of reasoning, one may further address, at least qualitatively, the melting the dust crystal. It is well known that the two-dimensional lattice melts in a hexatic phase [12]. Indeed, the oriented hexagonal plasma crystal can undergo a hexatic phase before the true three-dimensional melting as the lattice random motion intensifies [11,13,14]. Such intensified agitation of dust particles can result in weak horizontal bonds; furthermore, experiments [13] also show that the dust density hardly changes when the plasma approaches the solid-liquid transition. We therefore fix the lattice spacing and the total charge ratio between electrons 
and dust, and our solutions to eq. (3) for a given Mach number yield that the dust layer becomes significantly broadened and $\Delta \phi$ diminishes when $\kappa$ ( $\propto$ horizontal bond strength) decreases. It qualitatively describes how the weakening horizontal bonds in the hexatic phase should simultaneously lead to weak vertical bonds. Near the melting point, the negative "temperature" $\left(=-\kappa T_{\mathrm{e}}\right)$ can be close to zero. According to our results, the three-dimensional crystal at this point will have to melt since the vertical periodic structures can no longer exist, and the dust distribution will be the same as that of an isotropic fluid under the gravity. That is, the present model predicts a melting process consistent with the observation that somewhere in the hexatic phase, the plasma crystal should undergo a phase transition at the true three-dimensional melting point.

This work is supported in part by the National Science Council of Taiwan under the grants, NSC-87-2112-M008-009, NSC-87-2112-M008-010 and NSC-88-2112-M008-039. We thank LiN I and J. M. LIU for helpful discussions.

\section{REFERENCES}

[1] Chu J. H. and Lin I, Physica A, 205 (1994) 183; Chu J. H. and Lin I, Phys. Rev. Lett., 72 (1994) 4009; Chu J. H., Du J. B. and Lin I, J. Phys. D, 27 (1994) 296.

[2] Hayashi Y. and Tachibana K., Jpn. J. Appl. Phys., 33 (1994) L804.

[3] Thomas H., Morfill G. E., Demmel V., Goree J., Feuerbacher B. and Mohlman D., Phys. Rev. Lett., 73 (1994) 652.

[4] Melzer A., Trotenberg T. and Piel A., Phys. Lett. A, 191 (1990) 301.

[5] Hansen J. P., Phys. Rev. A, 8 (1973) 3096; Pollock E. L. and Hansen J. P., Phys. Rev. A, 8 (1973) 3110.

[6] Lee H. C., Chen D. Y. and Rosenstein B., Phys. Rev. E, 56 (1997) 4596.

[7] Vladimirov S. and Nambu M., Phys. Rev. E, 52 (1995) R2172.

[8] Melandsoe F. and Goree J., Phys. Rev. E, 52 (1995) 5312.

[9] Melzer A., Schweigert V. A., Schweigert I. V., Homann A., Peters S. and Piel A., Phys. Rev. E, 54 (1996) R46.

[10] Melandsoe F. and Goree J., J. Vac. Sci. Technol. A, 14 (1996) 511.

[11] Melandsoe F., Phys. Rev. E, 55 (1997) 7495.

[12] Thomas H. M. and Morfill G. E., J. Vac. Sci. Technol. A, 14 (1996) 501.

[13] Lin I, Juan W. T., Chiang C. H. and Chu J. H., Science, 272 (1996) 1626.

[14] Halperin B. I. and Nelson D. R., Phys. Rev. Lett., 41 (1978) 121; Young A. P., Phys. Rev. $B, 19$ (1979) 1855. 